\title{
Height effect on nucleation-site activity and size-dependent bubble dynamics in microchannel convective boiling
}

\author{
Man Lee ${ }^{1}$, Luthur Siu Lun Cheung ${ }^{2}$, Yi-Kuen Lee ${ }^{1}$ \\ and Yitshak Zohar ${ }^{2}$ \\ ${ }^{1}$ Department of Mechanical Engineering, Hong Kong University of Science and Technology, \\ Clear Water Bay, Kowloon, Hong Kong SAR, People's Republic of China \\ ${ }^{2}$ Department of Aerospace and Mechanical Engineering, University of Arizona, Tucson, \\ AZ, USA
}

Received 19 May 2005, in final form 13 July 2005

Published 3 October 2005

Online at stacks.iop.org/JMM/15/2121

\begin{abstract}
Forced convection boiling in microchannels is studied experimentally under the uniform heat flux boundary condition. Several microchannel heat sinks with integrated temperature sensors, spanning two orders of magnitude in height 5-500 $\mu \mathrm{m}$, have been fabricated with designed nucleation sites on the bottom surfaces. The microchannels are capped by a glass wafer to monitor bubble activity using video microscopy. Distributed micro heater elements on the device backside are used as the heat source, while the working liquid flow rate is adjusted using a syringe pump. The boiling curves of the device temperature as a function of the input power have been measured for various flow rates. The curves for increasing and decreasing heat flux exhibit a hysteresis loop, while the conditions corresponding to the onset of nucleate boiling and critical heat flux (CHF) are clearly distinguishable. The activity of nucleation sites as well as the ensuing bubble dynamics, from incipience to departure, is found to depend on the channel height. The critical size above which a nucleation site is active, along with three aspects of bubble dynamics, namely growth rate, departure size and release frequency, have been characterized experimentally and proper control parameters have been identified.
\end{abstract}

(Some figures in this article are in colour only in the electronic version)

\section{Introduction}

Two-phase convective flow in microchannels has become an active research topic in recent years for effective heat dissipation, especially in the cooling of electronic components. Since the heat capacity of liquid is normally higher than that of air, it has been suggested to use forced liquid flow. Various designs of microchannel heat sinks have been proposed to solve the cooling problem for the next generation of computer chips. The advantage of two-phase flow lies in the utilization of latent heat absorbed by the working fluid, due to the phase change from liquid to vapour, without temperature increase. Moreover, the temperature gradient along a microchannel in two-phase flow is smaller than in single-phase convective flow.
Bubble activity is an important heat transfer mechanism in convective boiling [1]. However, microchannel surfaces are usually too smooth to have adequate cavities for bubble nucleation. This could result in homogenous nucleation boiling, which requires a high temperature to trigger the phase change. The first two-phase flow mode in macroscale convective boiling is subcooled nucleate boiling [2]. Hsu [3] presented the first theoretical model to provide a quantitative criterion for predicting the size of an active nucleation site in pool boiling, while Davis et al [4] theoretically derived the condition of a critical radius for a nucleation site to be active in forced convection boiling. Peng et al [5] reported fully developed nucleate boiling in a microchannel with a hydraulic diameter of $650 \mu \mathrm{m}$. Jiang et al $[6,7]$ observed 


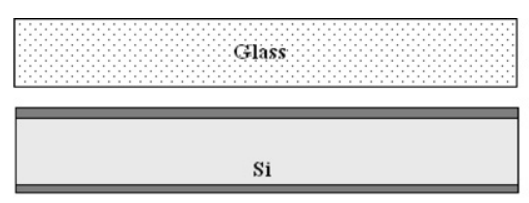

(a)

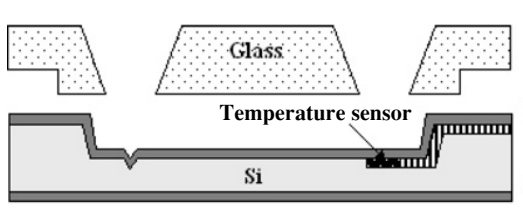

(c)

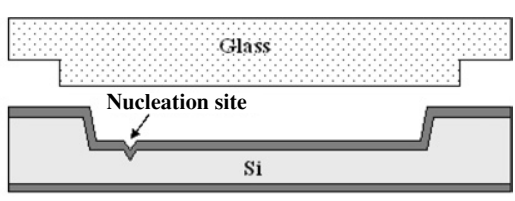

(b)

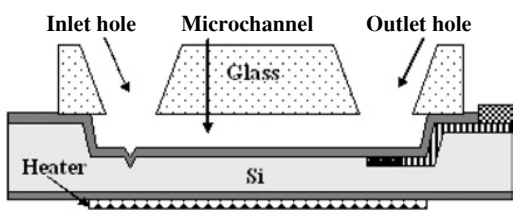

$(d)$

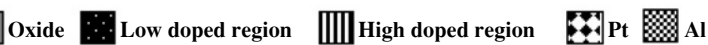

Figure 1. The major fabrication steps of the microchannel heat sink.

experimentally local nucleation boiling with bubble formation in microchannels with a hydraulic diameter as small as $26 \mu \mathrm{m}$. Hetsroni et al [8] and $\mathrm{Wu}$ et al [9] reported that the initial bubble activity typically took place near the microchannel outlet. Lee et al [10] documented the bubble formation and dynamics around certain active nucleation sites in a $14 \mu \mathrm{m}$ high microchannel. However, the size and location of the active nucleation sites were rather random. Zhang et al [11] created bottom cavities, $6 \mu \mathrm{m}$ in diameter and $200 \mu \mathrm{m}$ deep, to enhance bubble activity in a microchannel resulting in reduction of the superheat level.

The phase-change phenomenon in microchannels has indeed been discussed in several reports; however, studies of microscale bubble dynamics, including growth rate, departure size and release frequency, are still scarce. Surface forces in microsystems become dominant compared to body forces such as buoyancy, which is an important physical mechanism in macroscale bubble dynamics. Hence, although bubble nucleation has been observed in microchannels, microscale bubble dynamics could be very different. In macroscale forced convection boiling, Levy [12] developed a model to predict the bubble departure size based on the force balance between buoyancy, surface tension and flow drag. Thorncroft et al [13] reported that the bubble diameter increased with time following a $1 / 3$ power law, while the bubble departure diameter increased with temperature and decreased with increasing mass flux. Ma et al [14] found that the bubble diameter also increased with time following a $1 / 3$ power law, while the release frequency increased with increasing Reynolds number. In microscale forced convection boiling, Lee et al [15] investigated bubble dynamics in a microfabricated channel, about $40 \mu \mathrm{m}$ in hydraulic diameter, with relatively smooth walls. The experimental measurement of the bubble growth rate, departure size and release frequency was quite scattered.

In this work, a series of integrated microchannel heat sinks have been fabricated, with well-controlled nucleation sites at the channel bottom, to study the height effect on the activity of the nucleation site using various liquids as working fluids. Furthermore, methanol is used to explore size effects on bubble dynamics in microchannel forced convection boiling.

\section{Device design and fabrication}

Microchannel heat sinks, with nucleation sites at the channel bottom, have been designed to study the size effect on nucleation-site activity and bubble dynamics. The micro heat sinks, consisting of ten parallel microchannels, integrated with temperature sensors and heater elements have been fabricated on a silicon wafer using standard micromachining techniques. The channels, each $L=20-30 \mathrm{~mm}$ in length and $W=150$ $9000 \mu \mathrm{m}$ in width, vary in height in the range $H=5-510 \mu \mathrm{m}$. The grooves in a silicon substrate are capped by anodically bonding a glass wafer to allow monitoring of two-phase flow patterns. Nucleation sites are etched at the bottom of the microchannels; each site is shaped as an inverted pyramid with a square base, typical to the anisotropic TMAH etch of a (1 000$)$ silicon wafer. Hence, the characteristic scale of a nucleation site, $R$, is defined as twice the base area, $A_{\mathrm{b}}$, divided by its perimeter, $L_{\mathrm{b}}$, which is similar to the hydraulic radius concept, i.e. $R=2 A_{\mathrm{b}} / L_{\mathrm{b}}$. The fabricated nucleation sites range from $R=2.5$ to $370 \mu \mathrm{m}$ in size. Temperature microsensors are realized by the selective ion implantation of phosphorus on the microchannel bottom surface, while Pt micro heaters are fabricated on the silicon wafer backside to provide heat for bubble incipience.

The cross-sectional diagrams of the main fabrication steps are illustrated in figure 1 . The fabrication starts with wet thermal oxidation of a $0.3 \mu \mathrm{m}$ thick oxide layer on a $\left(\begin{array}{lll}1 & 0 & 0\end{array}\right)$ p-type Si wafer, $100 \mathrm{~mm}$ in diameter and $525 \mu \mathrm{m}$ in thickness, as shown in figure $1(a)$. The microchannel is patterned and formed by silicon etching using $25 \mathrm{wt} \%$ aqueous TMAH solution at $85{ }^{\circ} \mathrm{C}$. The nucleation sites, located at the bottom of the microchannels, are patterned by thick photoresist and etched by TMAH solution as shown in figure $1(b)$. The temperature sensing elements are lightly doped silicon regions created using phosphorus implantation with the dosage of $5 \times 10^{14} \mathrm{~cm}^{-2}$ and an implant energy of $60 \mathrm{keV}$. The sensor leads are heavily doped silicon regions formed by phosphorus diffusion at $1000{ }^{\circ} \mathrm{C}$ for $30 \mathrm{~min}$ as shown in figure $1(\mathrm{c})$.

The contact holes are then opened and a $1 \mu \mathrm{m}$ thick aluminium layer is sputtered and patterned to form the bonding pads for the temperature sensors. The Pt heater is made on the backside of the silicon substrate using a lift-off process 


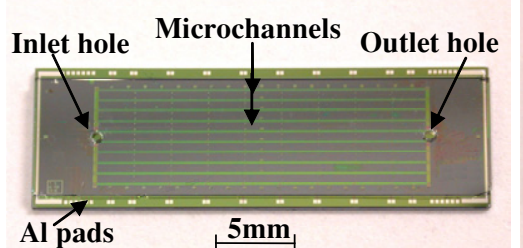

(a)

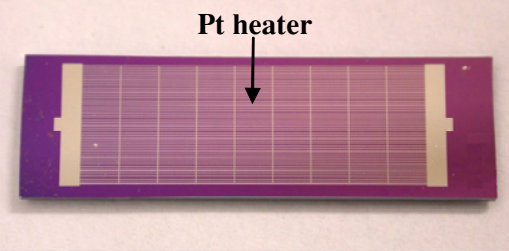

(b)

Figure 2. Pictures of a fabricated microchannel heat sink: $(a)$ the ten-microchannel array with inlet/outlet holes on the front side and (b) the Pt heater on the device backside.

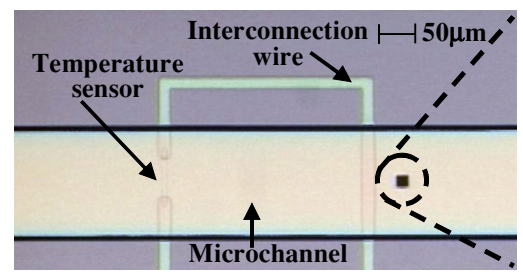

(a)

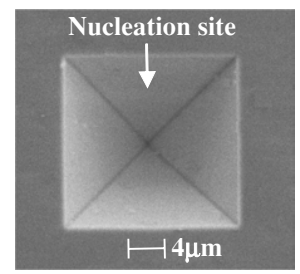

(b)
Figure 3. (a) A picture of a temperature sensor and a nucleation site inside a fabricated microchannel and $(b)$ a close-up SEM micrograph showing details of the nucleation site.

as depicted in figure $1(d)$. In parallel, steps, $6 \mu \mathrm{m}$ deep, are etched on Pyrex 7740 glass wafers by $49 \%$ HF to avoid direct contact between the aluminium layer and the cover glass as shown in figure $1(b)$. Next, inlet/outlet holes are drilled through the glass as shown in figure $1(c)$. Finally, the silicon and glass substrates are aligned and anodically bonded at $330{ }^{\circ} \mathrm{C}$ under an applied voltage of $700 \mathrm{~V}$. The glass covers are cut to expose the metal pads for subsequent wire bonding as demonstrated in figure $1(d)$. The front- and backside pictures of a fabricated device are respectively shown in figures 2(a) and $(b)$. The close-up pictures of a temperature sensor and a nucleation site, distributed along the microchannels, are shown in figures 3(a) and $(b)$, respectively. The interconnections are buried in the silicon substrate crossing the microchannels to contact the temperature sensors.

\section{Experimental arrangements}

The experimental set-up is schematically illustrated in figure 4; it consists of a syringe pump, pressure gauge, power supply, data acquisition system, CCD camera and DVD recorder. The working fluid is forced through the microchannels at a rate regulated by the constant displacement syringe pump, while the input power is controlled by adjusting the voltage difference applied across the heater. The inlet pressure is monitored by the pressure gauge. The fluid exiting the micro heat sink is cooled and condensed by passing it through cold water. The condensed fluid is collected in a graduated cylinder to measure the volume flow rate. The temperature sensors output signals are recorded using a computerized data acquisition system and, simultaneously, the evolving flow patterns in the microchannels are recorded through a CCD camera mounted on a microscope. The instantaneous bubble size is determined from the digital video, captured during the experiments, using a Matlab program.

Following the procedure described by Jiang et al [16], the temperature microsensors are calibrated prior to the experiments; each device is placed in an oven, and the resistance change of all sensors is recorded as a function of the controlled temperature change. The accuracy of temperature measurements is estimated to be within $\pm 0.1{ }^{\circ} \mathrm{C}$.

\section{Boiling curves}

The boiling curves were established by increasing the input power $q$ gradually and recording the corresponding device wall temperature, $T_{\mathrm{w}}$, while keeping the working fluid flow rate $Q$ constant. The experiments were repeated for micro heat sinks with the channel heights of 5,10 and $20 \mu \mathrm{m}$ with the same channel width of $150 \mu \mathrm{m}$ under different water flow rates. The results for the three devices under a constant water

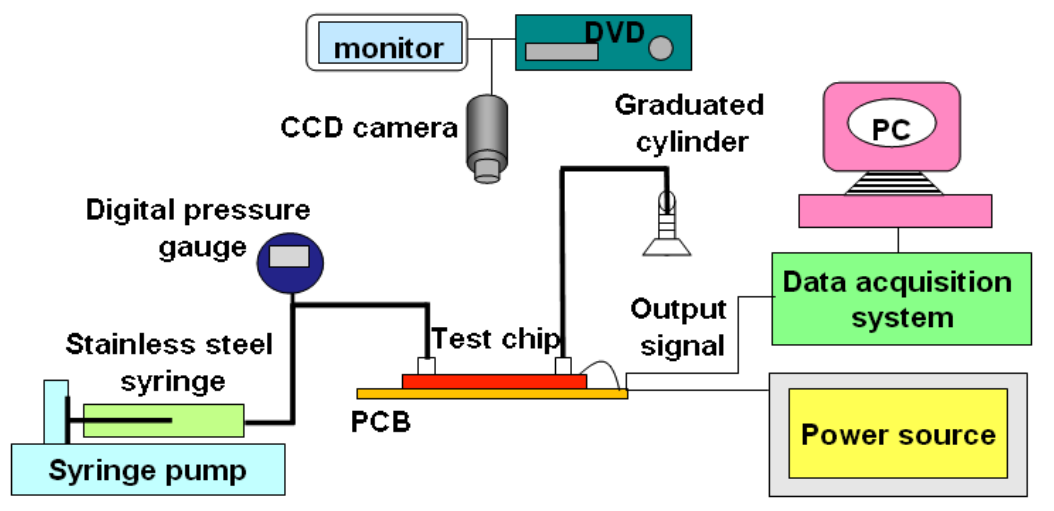

Figure 4. A schematic illustration of the experimental set-up. 


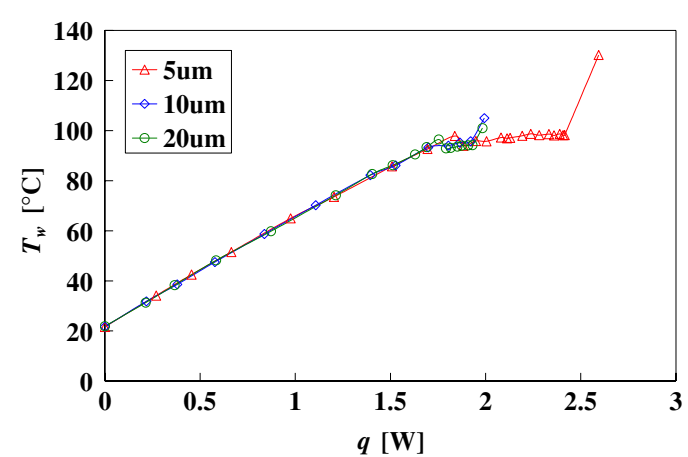

Figure 5. Boiling curves of the device wall temperature, $T_{\mathrm{w}}$, as a function of the input power, $q$, for the micro heat sinks 5, 10 and $20 \mu \mathrm{m}$ in channel height under the constant water flow rate of $Q=0.8 \mathrm{ml} \mathrm{h}^{-1}$.

flow rate of $Q=0.8 \mathrm{ml} \mathrm{h}^{-1}$ are summarized in figure 5 . Initially, the temperature increases linearly with the input power due to single-phase forced convection. The three curves are about the same since the slope is inversely proportional to the water flow rate. This functional relationship is identical for both increasing and decreasing input power as long as the flow is in the liquid phase. However, when two-phase flow develops, the boiling plateau is observed and the ensuing flow behaviour depends on the microchannel size. The input power to trigger the onset of the critical heat flux (CHF) condition for the microchannel height of $5 \mu \mathrm{m}, 10 \mu \mathrm{m}$ and $20 \mu \mathrm{m}$ is $2.4 \mathrm{~W}, 1.9 \mathrm{~W}$ and $1.9 \mathrm{~W}$, respectively.

An interesting hysteresis loop, also reported in macroscale forced convection [17-19], occurs for a higher input-power range with the development of two-phase flow as detailed in figure $6(a)$ for the $10 \mu \mathrm{m}$ high device and the water flow rate of $3.1 \mathrm{ml} \mathrm{h}^{-1}$. The initial deviation from linearity occurs at point A, corresponding to the onset of the phase change at the device outlet. The slope of the boiling curve, therefore, decreases slightly as a fraction of the added heat flux is converted into latent heat. The microchannel flow continues as single-phase liquid up to point $\mathrm{B}$, where the phase change appears at the device inlet as well. As the heat flux increases between points $\mathrm{A}$ and $\mathrm{B}$, the vapour content in the intermittent exit flow increases while the flow through all the microchannels up to the device outlet is still in the liquid phase. At point B, a burst of vapour emanating from the device inlet sweeps through the microchannels, as demonstrated in figure $7(a)$, triggering

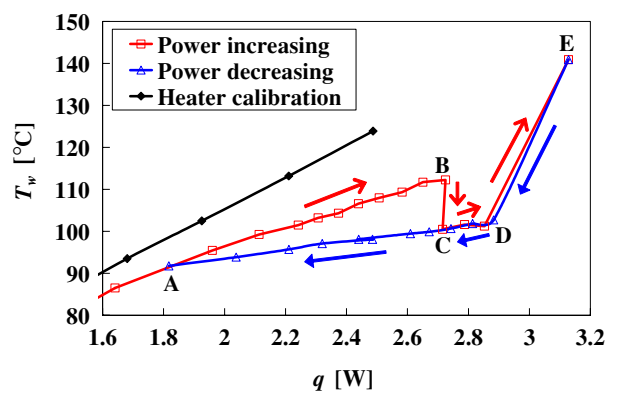

(a)

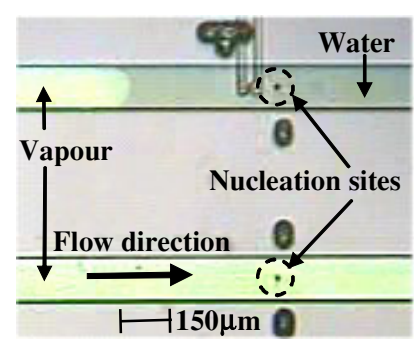

(a)

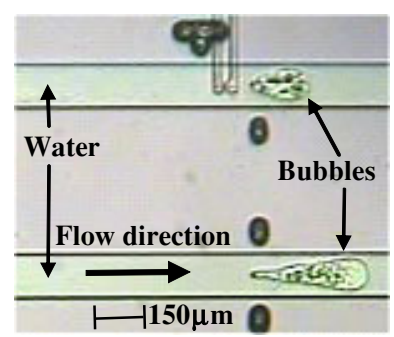

(b)
Figure 7. Pictures of the flow pattern at nucleate-boiling onset: (a) vapour burst from the inlet sweeps through the microchannels (point $\mathrm{B}$ in figure $6(a)$ ) and $(b)$ active nucleation sites triggered by the vapour (point $\mathrm{C}$ in figure $6(a)$ ).

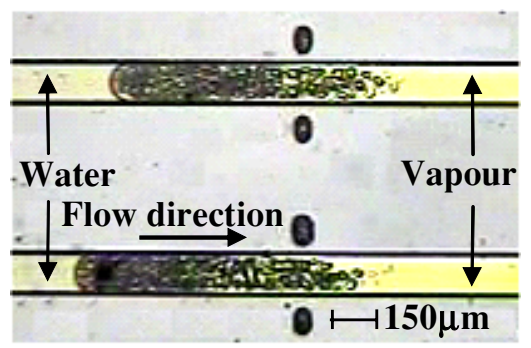

Figure 8. A picture of the flow pattern at the onset of the CHF condition (point D in figure 6(a)) showing the transition from liquid to vapour phase flow near the micro heat sink inlet.

the onset of nucleate boiling. This process is accompanied by a sharp drop in the temperature, from point $B$ to $C$, due to the latent heat required for bubble formation since some nucleation sites inside the microchannels become active as shown in figure $7(b)$.

Further increase in input power, between points $\mathrm{C}$ and $\mathrm{D}$, results in higher vapour content in the microchannels with almost constant temperature. Consequently, the transition region from the upstream single-phase liquid to the downstream single-phase vapour is continuously shifted upstream. Once the liquid-to-vapour transition region nears the device inlet, as in figure 8 , a critical heat flux (CHF) condition develops followed by a sharp temperature increase from point $\mathrm{D}$ to $\mathrm{E}$ as a result of dry out. Then, the entire flow in the microchannels, from the device inlet to the outlet, is in single-phase vapour. The boiling curve follows a different path E-D-C-A rather than E-D-C-B-A, figure 6(a), upon

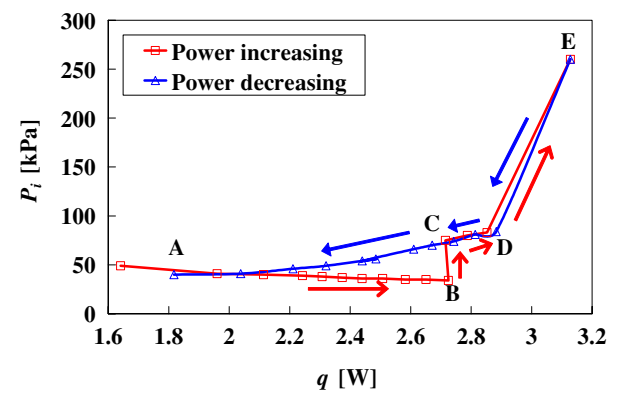

(b)

Figure 6. The hysteresis loop in the boiling curve in terms of the $(a)$ wall temperature, $T_{\mathrm{w}}$, and $(b)$ inlet pressure, $P_{\mathrm{i}}$, as a function of both increasing and decreasing input power, $q$, for the $10 \mu \mathrm{m}$ high microchannel heat sink under the constant flow rate of $Q=3.1 \mathrm{ml} \mathrm{h}-1$. 


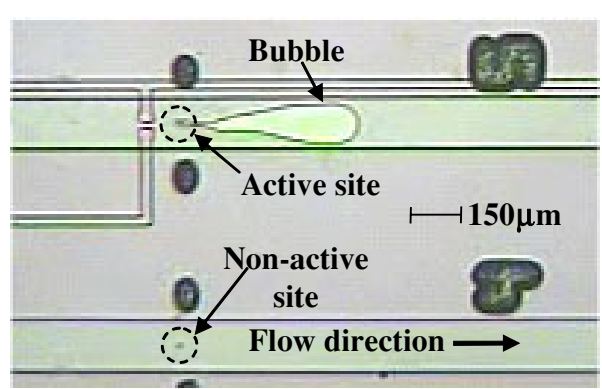

Figure 9. A picture showing the active $(R=4 \mu \mathrm{m})$ and non-active $(R=3.3 \mu \mathrm{m})$ nucleation sites in the micro heat sink with microchannels $5 \mu \mathrm{m}$ in height $\left(Q=0.8 \mathrm{ml} \mathrm{h}^{-1}\right.$ and $\left.q=1.75 \mathrm{~W}\right)$.

decreasing the input power from the dry-out state at point E. This is due to the continuous bubble activity at the nucleation sites during the input-power decrease between points $\mathrm{C}$ and $\mathrm{A}$. Hence, the temperature along the path $\mathrm{C}-\mathrm{A}$ is smaller than the path $\mathrm{A}-\mathrm{B}$, along which the flow is in single-phase liquid with no bubble activity.

A similar hysteresis loop is observed in figure $6(b)$ when plotting the inlet pressure gauge, $P_{\mathrm{i}}$, rather than the device temperature, as a function of the input power. The initial inlet pressure required to maintain the $3.1 \mathrm{ml} \mathrm{h}^{-1}$ constant flow rate under zero input power is $140 \mathrm{kPa}$. The inlet pressure decreases monotonically with increasing input power through points $\mathrm{A}$ and $\mathrm{B}$ as the device temperature increases and, hence, the liquid viscosity decreases. Since the outlet pressure is constant (atmospheric) and the flow rate is kept constant by the syringe pump, the inlet pressure decreases in order to maintain the same flow rate with lower fluid viscosity. When the onset of nucleate boiling at point B occurs, the pressure rises sharply from point $\mathrm{B}$ to $\mathrm{C}$ due to the increased volume associated with the phase change from liquid to vapour. Then, the inlet pressure increases gradually with increasing input power from point $\mathrm{C}$ to $\mathrm{D}$ because of the enhanced vapour content in the microchannels, while the onset of the critical heat flux condition is accompanied by a sharp rise in the inlet pressure from point $\mathrm{D}$ to $\mathrm{E}$. The inlet pressure also follows the path $\mathrm{E}-\mathrm{D}-\mathrm{C}-\mathrm{A}$ rather than $\mathrm{E}-\mathrm{D}-\mathrm{C}-\mathrm{B}-\mathrm{A}$ while reducing the input power from point $\mathrm{E}$. Along the path $\mathrm{C}-\mathrm{A}$, bubble activity is present and so the inlet pressure along $\mathrm{C}-\mathrm{A}$ has to be higher than that along $\mathrm{A}-\mathrm{B}$ to maintain the same water flow rate. Although the exact temperature and pressure values corresponding to points A-B-C-D-E in figure 6 fluctuate due to measurement errors, the hysteresis-loop phenomenon has repeatedly been observed in numerous experiments.

\section{Nucleation-site activity}

As the input power increases between the onset of nucleate boiling and the onset of CHF, only some but not all the fabricated nucleation sites become active as shown in figure 9 . There seems to be a critical size such that larger nucleation sites are active while smaller sites are inactive. Furthermore, under the same operating conditions of the flow rate and input power, the critical size is experimentally found to decrease with the channel height. For example, under $Q=0.8 \mathrm{ml} \mathrm{h}^{-1}$ and $q=1.75 \mathrm{~W}$, nucleation sites smaller than 3.3, 7.4 and $13.6 \mu \mathrm{m}$ are correspondingly inactive in the 5, 10 and $20 \mu \mathrm{m}$ high microchannel heat sinks, while sites larger than 4, 8 and $16.7 \mu \mathrm{m}$ are active. Changing the input power and water flow rate, within the limited tested range, seems to have no effect on the nucleation-site activity. This is in contrast with a critical size for a nucleation site to be active, $R_{\mathrm{t}}$, derived theoretically for flow boiling over a solid wall in a semi-infinite domain [4]. The derivation is based on the assumptions of the spherical bubble shape and conduction being the dominant heat-transfer mechanism at wall vicinity. Under the condition that the liquid at the outer edge of the bubble is just hot enough for the bubble to continue growing at an active nucleation site, the critical size is given by

$$
R_{\mathrm{t}}=\left(\frac{2 \sigma T_{\mathrm{s}} k_{1}}{q^{\prime \prime} h_{\mathrm{fg}} \rho_{\mathrm{g}}}\right)^{1 / 2}
$$

where $\sigma, T_{\mathrm{s}}, h_{\mathrm{fg}}, \rho_{\mathrm{g}}$ and $k_{1}$ are the surface tension, saturation temperature, latent heat, vapour density and liquid thermal conductivity of the working fluid, respectively, while $q^{\prime \prime}$ is the heat flux. The bubble nucleation activity in microscale, therefore, is different from the theoretical macroscale model.

In order to explore the size effect, the nucleation-site activity has been studied in eight microchannels ranging in height from 5 to $510 \mu \mathrm{m}$ with three different working fluids: ethanol, methanol and water. The dimensional analysis of the relevant variables yields two non-dimensional groups: $(R / H)$ and $\left(H \rho_{\mathrm{g}} h_{\mathrm{fg}} / \sigma\right)$. The activity of all fabricated nucleation sites under various operating conditions is summarized in figure 10 , using these two dimensionless parameters, where full symbols indicate active sites and hollow symbols indicate non-active sites. A sharp boundary between the active and the non-active sites is observed. The best curve fit to the boundary between the two regions yields an empirical formula for the nucleation site critical size $R_{\mathrm{c}}$ given by

$$
\frac{R_{\mathrm{c}}}{C_{1} H+C_{2}}=\left(\frac{H \rho_{\mathrm{g}} h_{\mathrm{fg}}}{\sigma}\right)^{n} .
$$

The constants are found to be $n=1 / 3$ with either $C_{1}=0.088$ and $C_{2}=0.268 \times 10^{-6} \mu \mathrm{m}$ for $5 \mu \mathrm{m} \leqslant H \leqslant 120 \mu \mathrm{m}$ or $C_{1}=$ 0.02 and $C_{2}=8.52 \times 10^{-6} \mu \mathrm{m}$ for $120 \mu \mathrm{m}<H \leqslant 510 \mu \mathrm{m}$. Thus, nucleation sites with size $R>R_{\mathrm{c}}$ are active, while sites with size $R<R_{\mathrm{c}}$ are inactive. This is a clear size effect, since the critical size increases with the microchannel height. Equation (2) is suitable for both working fluids ethanol and methanol as shown in figures $10(a)$ and $(b)$, respectively. However, the nucleation-site activity in water does not follow equation (2) for the microchannel height larger than $200 \mu \mathrm{m}$ as shown in figure $10(c)$. Surprisingly, in repeated experiments, no active nucleation sites have been observed in microchannels varying in height from 200 to $510 \mu \mathrm{m}$ although the fabricated cavity size range is very wide, $33 \mu \mathrm{m} \leqslant R \leqslant 370 \mu \mathrm{m}$. Perhaps, the combination of the nucleation-site geometry (size and shape) and water physical properties (latent heat and surface tension) prevents vapour entrapment, which is a prerequisite condition for bubble incipience. Nevertheless, the observed critical size values for the three working fluids are well correlated with the single curve calculated based on equation (2) as shown in figure 11.

\section{Microscale bubble dynamics}

An active nucleation site features a periodic process of bubble formation, growth and departure. The evolution of a bubble 


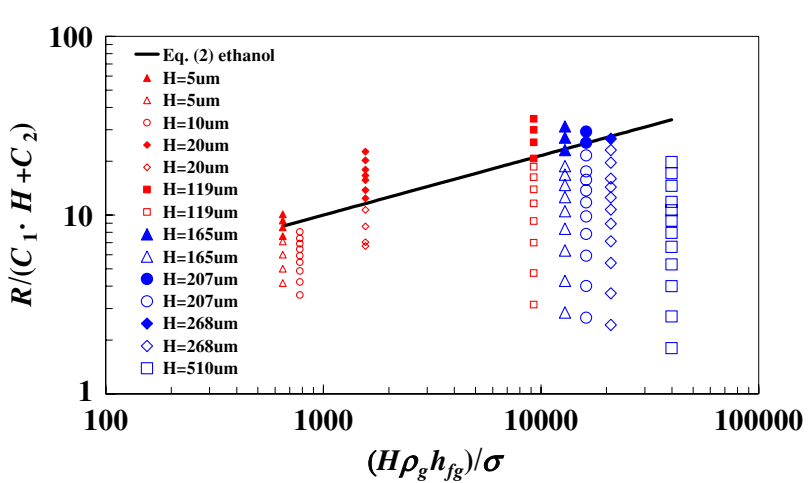

(a)

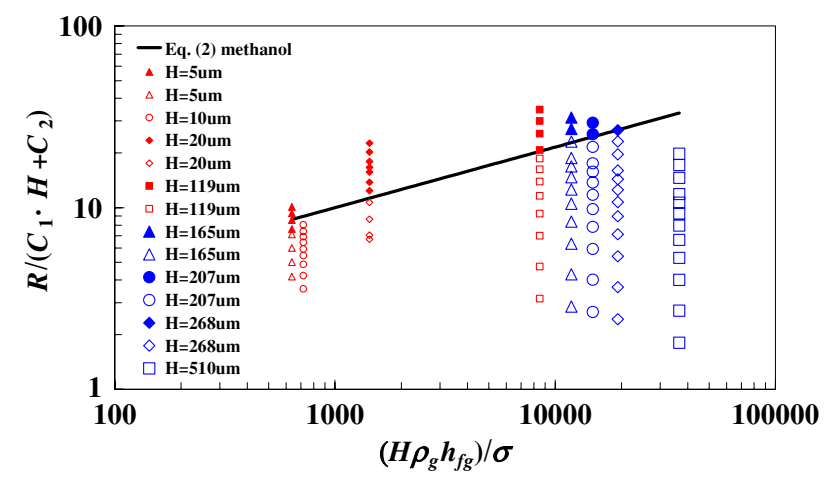

(b)

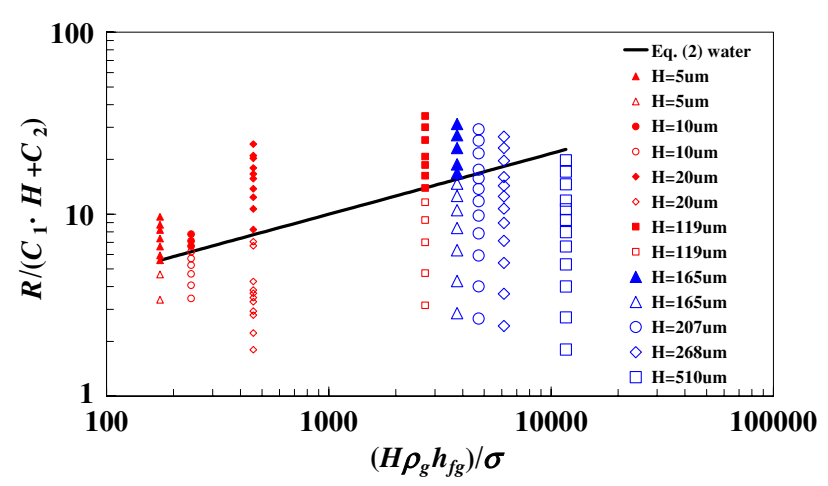

(c)

Figure 10. The microchannel-height effect on the nucleation-site activity for $(a)$ ethanol, $(b)$ methanol and $(c)$ water as the working fluid; full symbols denote active sites and hollow symbols inactive nucleation sites.

from its incipience to departure is termed bubble dynamics, which is characterized by three parameters: bubble growth rate, departure size and release frequency. Micro heat sinks with 5 and $10 \mu \mathrm{m}$ channel heights are utilized to characterize bubble dynamics using methanol as the working fluid. A typical cycle in a $10 \mu \mathrm{m}$ high microchannel is illustrated in the picture series depicted in figure 12 under a constant flow rate of $Q=1.6 \mathrm{ml} \mathrm{h}^{-1}$ and a steady input power of $q=0.4 \mathrm{~W}$. Once the critical conditions for bubble nucleation have been reached and maintained, the bubble evolution process becomes very periodic. The bubble is formed from a pre-existing nucleus within the fabricated nucleation site, figure 12(a). The bubble then grows steadily with the vapour phase occupying the space between the channel top and bottom surfaces,

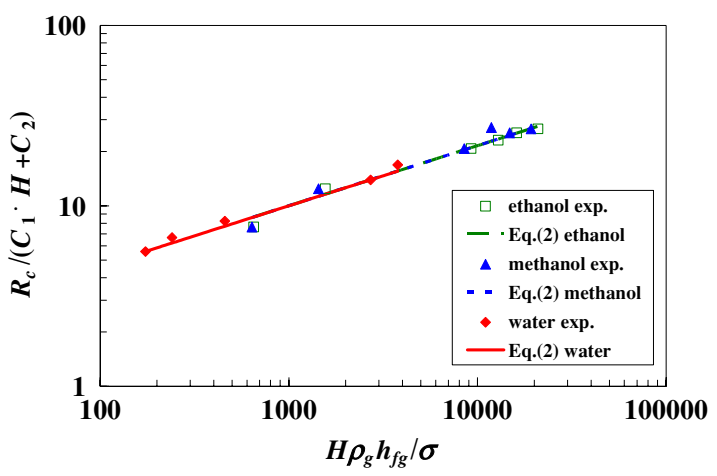

Figure 11. The correlation between the critical size for nucleation-site activity and the microchannel height for ethanol, methanol and water as working fluids.

figure $12(b)$, hence, resulting in moving contact lines. The bubble, anchored at the nucleation site, expands in the downstream direction, figure $12(c)$, due to the driving negative pressure gradient. The bubble finally departs, assuming a cylindrical shape when the pushing exceeds pulling forces, figure $12(d)$, thus allowing a new cycle to start.

\subsection{Bubble growth rate}

The instantaneous bubble planar area is directly measured from the extracted still pictures in order to estimate the bubble size. Assuming the bubble shape to be uniform across the microchannel height, the volume of the bubble, $V_{\mathrm{B}}$, is calculated as the product of the planar area and the channel height. The time-dependent volume of several successive bubbles, formed at the same nucleation site, is plotted in figure 13. The bubble size increases almost linearly with time, $t$, implying a constant growth rate; this is consistent with the macroscale linear bubble growth reported in other works $[13,14]$. Since the microchannel height is so small compared to the bubble size, the bubble comes in contact with the channel top surface at a very early state of its evolution restricting its growth in the cross-stream direction. Therefore, its shape is no longer spherical during the entire bubble dynamic process. Furthermore, the bubble is tilted in the streamwise direction, similar to the macroscale behaviour, resulting in the balloonlike shape due to the flow drag effect. However, the narrow gap between the top and bottom microchannel surfaces results in an essentially 2D shape rather than a 3D shape.

The bubble growth rate dependence on the input power, under a constant flow rate, is summarized in figure 14. The time-dependent bubble volume for a wide range of wall temperature, proportional to the input power, is plotted in figure 14(a). Clearly, the bubble departure size, the end point of each curve, is practically constant independent of the input power. However, at steady state, the vaporization rate increases with increasing heat flux. Hence, the bubble growth rate increases with increasing wall temperature (input power) as indeed shown in figure $14(b)$. This is consistent with macroscale forced convection boiling, in which the bubble growth rate increases with the input heat flux [14]. 


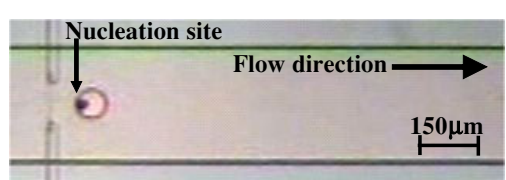

(a)

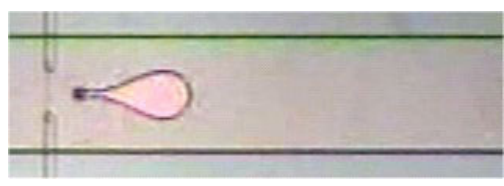

(c)

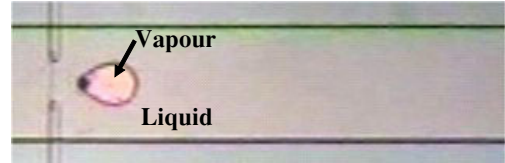

(b)

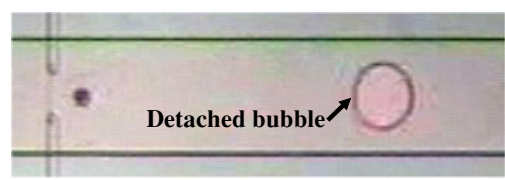

(d)

Figure 12. A series of still pictures extracted from a video clip of a complete bubble dynamics cycle from bubble incipience to departure in the $10 \mu \mathrm{m}$ high microchannel $\left(Q=1.6 \mathrm{ml} \mathrm{h}^{-1}\right.$ and $\left.q=0.4 \mathrm{~W}\right)$.

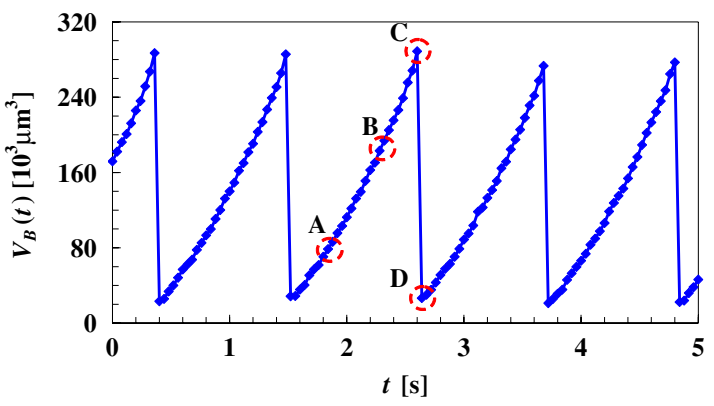

Figure 13. The time evolution of the bubble size for several successive cycles at $T_{\mathrm{w}}=49^{\circ} \mathrm{C}$ with $W / H=30$ and $R e=0.54$; the stages A-D correspond to the pictures in figures $12(a)-(d)$.

\subsection{Bubble departure size}

The flow drag force dominates bubble characteristics in a confined geometry, especially its departure size. On the verge of departure, shown in figure 15 , the bubbles assume a balloonlike shape connected to the nucleation site by a thin long tail; both the bubble shape and size are very repeatable, as shown in figure 13, under a constant flow rate and heat flux. However, the bubble departure size increases with decreasing flow rate as demonstrated in figure 15. This indicates that the Reynolds number, $R e=2 H \rho_{1} U / \mu$, is one of the control parameters for the bubble departure phenomenon; $\rho_{1}, \mu$ and $U$ are the liquid density, fluid viscosity and average velocity, respectively.

The bubble departure size dependence on both flow rate and wall temperature has been investigated. The measured volume of the bubble at departure, $V_{\mathrm{BD}}$, is plotted in

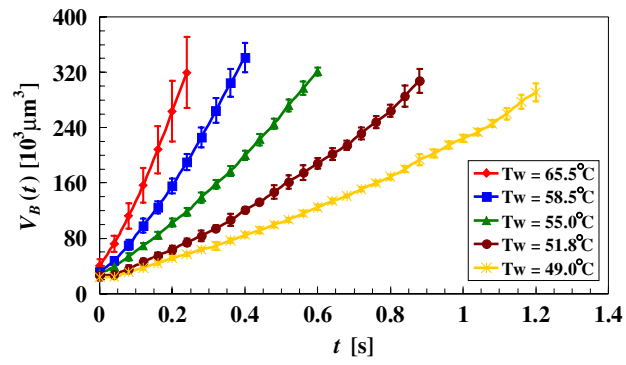

(a)

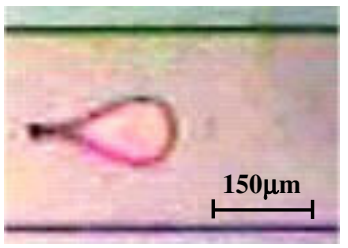

(a)

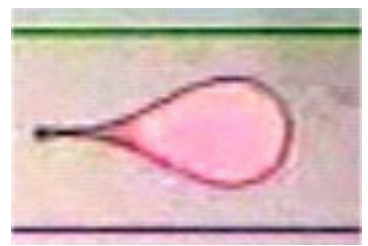

(b)
Figure 15. The Reynolds number effect on the bubble departure size at $T_{\mathrm{w}}=53{ }^{\circ} \mathrm{C}:(a) R e=0.1012$ and $(b) R e=0.0337$.

figure $16(a)$ as a function of the wall temperature (input power) for three flow rates (Reynolds numbers). Evidently, the bubble departure size is independent of the input power over a wide wall temperature range, $T_{\mathrm{w}}=50-66^{\circ} \mathrm{C}$, within an experimental error. However, increasing the Reynolds number results in a smaller bubble size at departure. In macrochannels, the bubble departure diameter increases with temperature and decreases with increasing mass flux [13].

In the absence of a proper mathematical model, dimensional analysis [20] has been utilized to explore the relationship between the bubble departure size and the Reynolds number. The channel height is assumed to be the only relevant length scale in the evolution of bubbles; hence, it is selected as the normalizing scale for the departure size. The normalized bubble departure size is plotted in figure $16(b)$ as a function of $R e$ with the wall temperature, $T_{\mathrm{w}}$, and channel aspect ratio, $W / H$, as varying parameters. All data collapse onto a single curve suggesting that, at least within the tested parameter space, the scaling choice

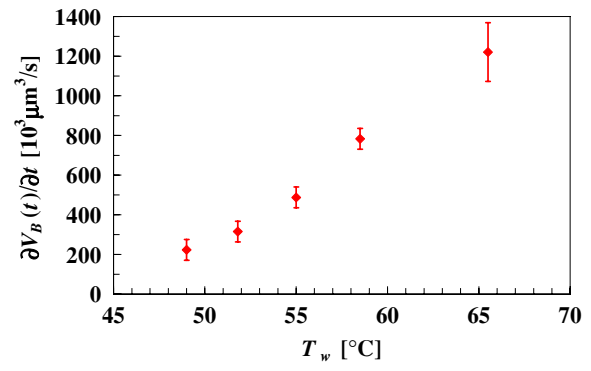

(b)

Figure 14. (a) Bubble volume (size) growth with time for various wall temperatures (input power); the maximum bubble size (at departure) is constant, and $(b)$ the average bubble growth rate as a function of the wall temperature. 


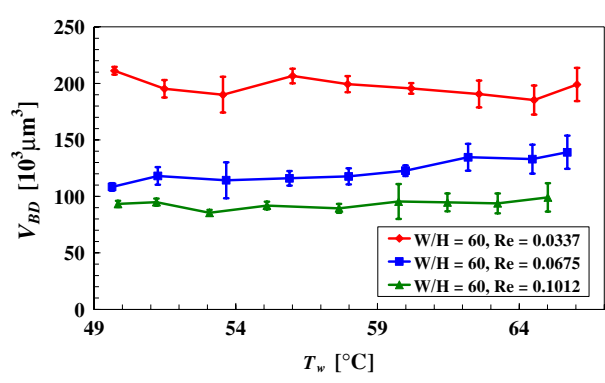

(a)

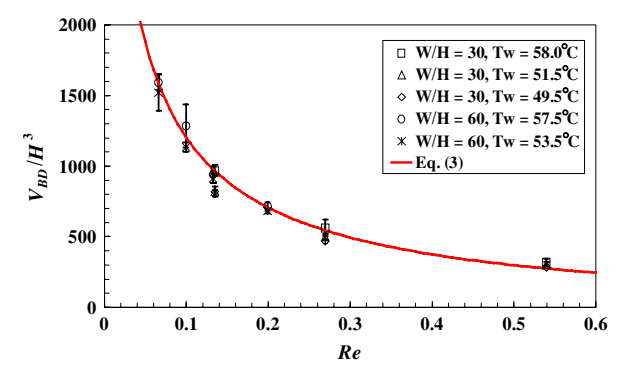

(b)

Figure 16. (a) The bubble departure size dependence on the wall temperature for various Reynolds number values and (b) normalized bubble departure size dependence on the Reynolds number.

is proper. The non-dimensional bubble departure size is found to decrease exponentially with increasing Reynolds number and, utilizing a curve-fitting technique, an empirical formula has been deduced as follows:

$$
\frac{V_{\mathrm{BD}}}{H^{3}}=2 \times 10^{4} \exp \left(-5 R e^{0.25}\right) \text {. }
$$

Thus, in microchannels, the bubble departure volume $V_{\mathrm{BD}}$ is determined by the channel height and Reynolds number. The bubble departure diameter in macroscale convective boiling, $D_{\mathrm{d}}$, is approximately given by [12]

$$
D_{\mathrm{d}} \propto \sqrt{\frac{\sigma}{g\left(\rho_{\mathrm{l}}-\rho_{\mathrm{g}}\right)+C \tau_{\mathrm{w}} / H_{\mathrm{b}}}}
$$

where $\tau_{\mathrm{w}}$ and $H_{\mathrm{b}}$ are the wall shear stress and bubble height, respectively, while $C$ is a constant coefficient. The wall shear stress is proportional to the square of the flow rate. Thus, in macrochannels, the bubble departure size is mainly the result of a balance between buoyancy, surface tension forces and the flow drag. In microchannels, however, buoyancy is negligible as bubbles fill up the entire space between the top and bottom surfaces, and their motion is confined to the downstream direction only. Hence, bubbles in microchannels depart from nucleation sites when the flow drag force, depending only on the Reynolds number and the bubble size, is sufficiently high. The role of surface tension has not been investigated, and it could have similar effect to that found in macrochannels.

\subsection{Bubble release frequency}

The time-evolution traces of the bubble size, such as the example in figure 13 , can also be used to estimate the number of bubbles formed at a particular nucleation site per unit time, i.e. bubble release frequency, $f$. The bubble release frequency under varying combinations of flow rate, input power and channel dimensions has been measured; it is found to be the most sensitive to input power. Increasing the heat flux increases not only the wall temperature but also the vaporization rate. Therefore, the bubble release frequency is plotted in figure 17 as a function of the wall temperature for various conditions. All the results seem to follow a similar trend and, utilizing the curve-fitting method, the following exponential relationship has been found:

$$
f^{*}=0.0013 \exp \left(3 \times 10^{-5} \theta\right)
$$

where the dimensionless release frequency, $f^{*}$, and wall temperature, $\theta$, are defined as

$$
f^{*}=f W^{2} \rho_{1} / \mu
$$

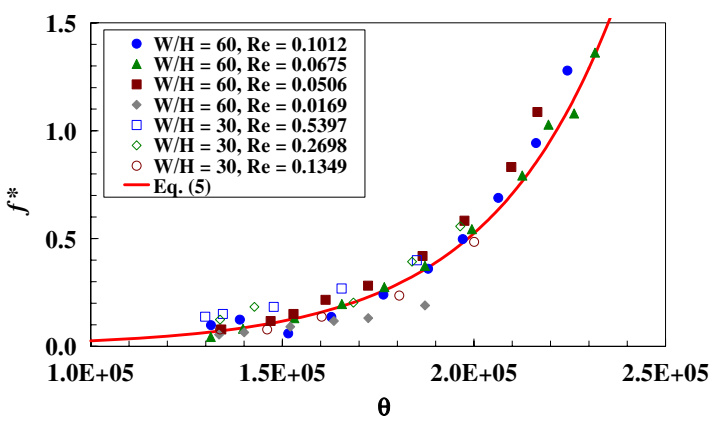

Figure 17. The normalized bubble release frequency dependence on the non-dimensional wall temperature.

and

$$
\theta=\left(T_{\mathrm{w}}-T_{\infty}\right) W k_{1} \rho_{\mathrm{l}} /(\mu \sigma)
$$

where $T_{\infty}$ is the ambient temperature. Since the bubble growth rate increases while its departure size stays constant with increasing input power, the bubble lifetime is shorter. This means a higher bubble release frequency, if there is no time delay between the departure of an old bubble and the formation of a new one, as has indeed been observed. In macroscale pool boiling, the bubble release frequency is correlated with its departure size as $f \cdot\left(D_{\mathrm{d}}\right)^{n}=$ const [21]. The relationship still holds in convective boiling if the bubble size is much smaller than the channel characteristic scale. However, in microchannels, the bubble departure size is constant while the release frequency increases with the input power.

\section{Conclusions}

A set of microchannel heat sinks, integrated with temperature sensors, varying in channel height from 5 to $510 \mu \mathrm{m}$ has been fabricated for the study of forced convection boiling in microchannel flow. Nucleation sites, shaped as an inverted pyramid with a square base, have been etched at the channel bottom with a characteristic size ranging between 2.5 and $370 \mu \mathrm{m}$.

The boiling curves are initially linear as the entire flow is in single-phase liquid. In the higher input-power range, the boiling curve features a hysteresis loop. Phase change starts at the device outlet and, with increasing heat flux, appears at the inlet. At a certain stage, a burst of vapour sweeps through the microchannels triggering the onset of nucleate boiling. 
Further increase of the input power results in a critical heat flux condition, where the entire microchannel flow is in singlephase vapour.

Bubble nucleation activity is found to depend on the channel height using three different working fluids: water, methanol and ethanol. An empirical formula for the critical size is suggested. The critical size, above which nucleation sites are active, increases with the channel height. Hence, smaller nucleation sites are active in smaller height microchannels.

Bubble dynamics in microchannels have been studied experimentally in $5 \mu \mathrm{m}$ and $10 \mu \mathrm{m}$ high microchannels. The bubbles, practically two-dimensional, assume a balloon-like shape elongated in the streamwise direction. The bubbles grow in volume essentially at a constant rate, which increases with increasing input power. The bubble departure size, independent of the input power, decreases exponentially with increasing Reynolds number (flow rate). The normalized bubble release frequency is found to increase exponentially with increasing wall temperature (input power).

\section{Acknowledgment}

This work is supported by Hong Kong Research Grants Council through grant HKUST6134/04E.

\section{References}

[1] Dhir V K 1991 Nucleate and transition boiling heat transfer under pool and external flow conditions Int. J. Heat Fluid Flow 12 290-314

[2] Collier J G and Thome J R 1994 Convective Boiling and Condensation (Oxford: Clarendon)

[3] Hsu Y Y 1962 On the size range of active nucleation cavities on a heating surface ASME J. Heat Transfer $\mathbf{8 4}$ 207-16

[4] Davis E J and Anderson G H 1966 The incipience of nucleate boiling in forced convection flow AIChE J. 12 774-80

[5] Peng X F and Wang B X 1993 Forced convection and flow boiling heat transfer for liquid flowing through microchannels Int. J. Heat Mass Transfer 36 3421-7
[6] Jiang L, Wong M and Zohar Y 1999 Phase change in microchannel heat sinks with integrated temperature sensors J. Microelectromech. Syst. 8 358-65

[7] Jiang L, Wong M and Zohar Y 2001 Forced convection boiling in a microchannel heat sink J. Microelectromech. Syst. 10 80-7

[8] Hetsroni G, Mosyak A, Segal Z and Pogrebnyak E 2003 Two-phase flow patterns in parallel micro-channels Int. J. Multiphase Flow 29 341-60

[9] Wu H Y and Cheng P 2003 Visualization and measurements of periodic boiling in silicon microchannels Int. J. Heat Mass Transfer 46 2603-14

[10] Lee M, Wong Y Y, Wong M and Zohar Y 2003 Size and shape effects on two-phase flow patterns in microchannel forced convection boiling J. Micromech. Microeng. 13 155-64

[11] Zhang L, Wang E N, Goodson K E and Kenny T W 2005 Phase change phenomena in silicon microchannels Int. J. Heat Mass Transfer 48 1572-82

[12] Levy S 1967 Forced convection subcooled boiling-prediction of vapour volumetric fraction Int. J. Heat Mass Transfer 10 951-65

[13] Thorncroft G E, Klausner J F and Mei R 1998 An experimental investigation of bubble growth and detachment in vertical upflow and downflow boiling Int. J. Heat Mass Transfer 41 3857-71

[14] Ma Y and Chung J N 2001 A study of bubble dynamics in reduced gravity forced-convection boiling Int. J. Heat Mass Transfer 44 399-415

[15] Lee P C, Tseng F G and Pan C 2004 Bubble dynamics in microchannels. Part I: single microchannel Int. J. Heat Mass Transfer 47 5575-89

[16] Jiang L, Wong M and Zohar Y 1999 Micromachined polycrystalline thin film temperature sensors Meas. Sci. Technol. 10 653-64

[17] Lazarek G M and Black S H 1982 Evaporative heat transfer, pressure drop and critical heat flux in a small vertical tube with R-113 Int. J. Heat Mass Transfer 25 945-59

[18] Yoon P H, Jeong J and Kang Y T 2004 Boiling hysteresis at low temperature on enhanced tubes Int. J. Refrig. 27 4-9

[19] Piasecka M, Hozejowska S and Poniewski M E 2004 Experimental evaluation of flow boiling incipience of subcooled fluid in a narrow channel Int. J. Heat Fluid Flow 25 159-72

[20] Taylor E S 1974 Dimensional Analysis for Engineers (Oxford: Clarendon)

[21] Ivey H J 1967 Relationships between bubble frequency, departure diameter and rise velocity in nucleate boiling Int. J. Heat Mass Transfer 10 1023-40 